\title{
Metadata Enhancement Through Name Authority in the UNT Digital Library
}

Hannah Tarver and Mark Phillips

\begin{abstract}
As a tool for the findability of general and special collections, descriptive metadata has played an increasingly important role for cultural heritage organizations, such as libraries, archives and museums around the world. As digital holdings grow, the opportunities to leverage this descriptive metadata have led these same organizations to develop and implement strategies to improve the quantity, quality and reusability of local metadata. The University of North Texas Libraries (UNTL) has worked on a number of fronts to make the descriptive metadata held and managed by the library a more valuable asset and to allow the use of descriptive metadata in new and interesting ways. Over the past three years, we have focused on controlling names in our system through iterative steps and the use of tools that make our name authority records as open and shareable as possible. Managing and leveraging metadata records and associated information will continue to grow in importance as the quantity of available records increases and provides new opportunities for discovery, aggregation and analysis; this will also encourage the development of workflows, tools and techniques to improve the quality, quantity and consistency of metadata records to support various functions. This chapter will serve as a case study discussing the UNTL's implementation of name authority control in the UNT Digital Library as a means of increasing metadata records' quality and consistency, and of improving end-user retrieval.
\end{abstract}

\section{Introduction}

Historically, libraries have focused not only on building collections, but on creating a means of describing these resources to make them discoverable and useful. Over time, cataloging -- or, more broadly, metadata creation -- has been standardized in various structured ways to ensure consistency at a local level and the ability to easily share resource descriptions among institutions. Within these structures, authority control functions as a key component for usability by enabling optimal search and retrieval of relevant resources by users. Simply defined, authority control is the process of identifying a single preferred or "authorized" format of a value that should be used in place of alternative spellings or synonyms to create consistent entries. There are a number of areas in which authority control is important for libraries including subjects, locations, and names of organizations, events, and persons.

Name authority tends to pose a particular problem because a metadata creator may need additional information associated with an authority heading to determine whether a name applies (particularly in the case of multiple persons with similar names). This may require a separate database of authority entries with contextual information, rather than a list of authorized terms or thesaurus, which are sufficient for some forms of authority control. Tillett (1989) notes that authority work generally involves research of names as well as documenting information in a name authority file such as "the authority data of preferred form, variants, history, scope, and links to other authority records." A number of initiatives in the library environment, both historically and in the present, have attempted to generate and share name authority records, and to address the challenge of keeping these systems up-to-date and relevant. Typically national libraries throughout the world take responsibility for names that are 
important to their national domain, including authors and organizations from their country that create resources of interest to their users. In the United States, this work has been carried out for decades by the Library of Congress (LOC) with their Name Authority File (LCNAF). Containing over eight million records, this dataset comprises names of people and organizations that appear in the LOC catalog.

In the past decade there has been an asserted effort to move name authority files from locally-managed library databases to the open web. For example, llik (2014) discusses the growing need to incorporate name authority sources and tools beyond traditional MARC records to better serve local needs. The goal is to make use of technologies such as Linked Open Data (LOD) in order to capitalize on the extensive name authority work we've already done in this space, so that it becomes more widely adoptable and usable by the rest of the web. The Library of Congress id.loc.gov system makes available a number of controlled vocabularies and name authority files as linked open data, which represents a major step toward open, shareable authority information in the United States. The Virtual International Authority File (VIAF) from OCLC furthers this work by aggregating name authority files from more than 24 national libraries and many other organizations around the world, matching name concepts that are the same, and making them available as linked data with established relationships between the different representations of the same entity (OCLC, 2015). VIAF has successfully generated and exposed LOD, and has been used on the web to help populate Wikipedia with name authority information (OCLC, December 2012). In the library sphere, VIAF has contributed to the work OCLC has done on its WorldCat product, which now provides millions of bibliographic records that are using the schema.org vocabularies (OCLC, June 2012).

\section{Academic names}

While these efforts represent a massive amount of work, communication, negotiation and finally agreement, they do not represent the full needs of academic institutions to manage identification of names within our systems. In 2009, Salo pointed out some of the difficulties in maintaining name authority in institutional repositories due to the varying sources of metadata, limitations of the systems and specificity of name authority systems. Many of the systems mentioned above are primarily populated with names and identities of notable people throughout history, politicians, performers, authors and artists. Furthermore, the authors represented in traditional name authority files tend to be solely those who have written books, rather than representing the wider array of scholarly publishing. No one organization can manage name authority files for all entities that might need to be represented in metadata records, however this means that large, established name authority files are suboptimal for managing the wide range of resources held at academic institutions. In particular, institutions that want to manage the scholarly research output of their faculty and staff may have difficulty managing names for persons who primarily publish scholarly or scientific articles, for which even widely-published authors may never receive a LCNAF record, nor make their way into VIAF.

In 2013 the University of North Texas (UNT) Libraries demonstrated this phenomena with research into the number of instances of name authority records that existed in local bibliographic records, VIAF, LCNAF, or in Wikipedia for 100 randomly-selected authors who had materials in the UNT Scholarly Works collection (UNT's institutional repository). This data is presented in Table 1. 


\begin{tabular}{|l|l|}
\hline UNT Scholarly Works Repository & 100 (random sampling) \\
\hline Local bibliographic records & 26 \\
\hline VIAF records & 32 \\
\hline LCNAF records & 28 \\
\hline Wikipedia & 2 \\
\hline
\end{tabular}

Table 1. VIAF, LOC, and Wikipedia Name Authority Record Comparison (Tarver, et al., 2013).

As evidenced by the data, only $27 \%$ of names are found in the local bibliographic records, $32 \%$ have an authority file in the VIAF database, $28 \%$ have an authority file in the LCNAF and only $2 \%$ of names were found in Wikipedia. Additionally, most authors with a record in one authority control file overlap with those controlled in another; therefore the total number of authors represented in all of these authority files is still roughly one-third of the total authors in the random sample. To control names for the full scope of resources in an academic library, we need a different approach to bridge this gap.

In the late 2000s a number of initiatives set out to help with this problem, such as Open Researcher and Contributor ID (ORCID) and International Standard Name Identifier (ISNI), which were created to help facilitate the creation of unique identifiers for all interested authors in the academic publication space. This work has been successful with over 1.3 million unique author identifiers being created in the ORCID system by 2015. These provide yet another source of identifiers and another tool for authority control (by using the identifier as the representation of the name in a linked data context) to manage names in the academic environment. Institutions such as Texas A\&M have begun the process of registering master's- and doctoral-level students with ORCID in order to establish unique identifiers for the students that they can use throughout their careers (Texas A\&M University Libraries, 2015). Additionally, many granting institutions and publishers now allow for primary investigators and authors to submit ORCIDs in addition to their names so that there is no question about which "John Smith" is publishing a paper or submitting a grant.

With all of these opportunities for names, one might think that there is a goldmine for metadata creators and system designers in the academic library context; however, great abundance does not eliminate all challenges. One challenge is the need to establish names for authors who are no longer living, but who were never assigned an authority record in a traditional or more recent system. Additionally, with millions of name authority records available, it is now possible to further confuse a metadata creator or end user by incorrectly selecting a record that appears correct but which might point to a different person with a similar name. A contrived example of this would be if there were two authors named Jane Smith who were publishing at the same time in similar-sounding fields, say linguistics and computational linguistics. With even more authority files in a variety of places, it is possible that having more authors named "Jane Smith" as options for metadata creation could create additional confusion. For instance, at the time of writing there are 3 ORCIDs for persons named "Mark Phillips," however, "0000-0002-9679-6730" is the identifier for the Mark Phillips involved in the writing of this chapter. 
While there is no single, perfect answer, we decided that the best option at UNT would be a locally-built name authority system which would let us manage locally-important names and also leverage existing name authority tools such as Wikipedia, ORCID, VIAF and LCNAF. This provides us the ability to improve not only the metadata creation process within academic libraries but also to improve the users' experiences with our systems.

\section{Background}

The UNT Libraries have been acquiring and hosting digital materials for more than ten years. Although all of the items reside in the same digital library infrastructure, they are searchable by end-users through three separate interfaces: The Portal to Texas History, the UNT Digital Library, and The Gateway to Oklahoma History. Over the years, we have recognized the importance of metadata in our Digital Collections and the potential for leveraging metadata values to support user interfaces and functionality. For example, when users search in one of the interfaces, the system automatically generates browseable facets based on values in several of our metadata fields including resource type, coverage place and language. Within individual records many values, such as names, locations and subjects, become clickable links that launch a search in our system for other items with the same value.

However, this dependence on metadata values requires a relatively high level of consistency across collections. All of the items in our collections are described using a locally-modified Dublin Core schema containing twenty-one available fields. Whenever possible, field values -- and all qualifiers -- are limited to controlled vocabulary options. Our vocabularies are maintained as RDF-compliant open linked data, which is connected to our metadata system so that vocabularies display as drop-down menus.

Additionally, we have extensive documentation detailing how metadata creators should format and enter information in each metadata field, with examples and resources (UNT Libraries, 2015).

In terms of name authority, we controlled names informally by encouraging the use of established authority records (including LCNAF and VIAF) when relevant. For names without authority records, we suggested general formatting guidelines, such as inverting personal names, using the fullest form of the name available (James rather than J.) and removing prefixes and suffixes except those necessary to identify an individual. We also attempted to use consistent formatting of each name within a collection of items. Although this helped to created general consistency within our records, there was still a need for more formal authority control, particularly within collections containing names associated with UNT. Many of the collections hosted in our system are owned and managed by partner institutions, so the amount of contextual information that we have for metadata or authority control is limited; however, names associated with UNT fall within the direct domain of our institution and are unlikely to be controlled by any other entity. It was still not always possible to find the most appropriate version of a name, or to verify that two similar names (e.g., Smith, D. and Smith, Don) were in fact the same person. These factors, in part, led to the development of the UNT Name App.

\section{UNT Name App}

The UNT Name App is a web-based application designed to allow us to locally manage name authority within our system. We had several goals, which included: making our work compatible with other name authority initiatives around the library world, working within the existing structures and best practices of Linked Open Data (LOD) and putting something in place that would require little work while still 
delivering a service that was usable in our local digital library system. To that end we decided to build a Django-based application written in Python that would act as both a website for our name authority service and as a set of application programming interfaces (APIs) that we could use in our digital library infrastructure.

We had several design goals in mind while building this tool. First, we decided that we wanted to map our work into the Metadata Authority Description Schema (MADS) standard. The Library of Congress used this vocabulary as the successor to MARC-based name authority records. However, we also wanted to work directly within the greater LOD community, so we used the core components of MADS as the primary data model and extended it to allow us to represent relationships with other existing identifiers around the web (see Table 2).

\begin{tabular}{|c|c|c|c|c|}
\hline Field & Required & Repeatable & Example & Notes \\
\hline Name ID & Yes & No & $\mathrm{nm0004212}$ & Created by the system \\
\hline $\begin{array}{l}\text { Authoritative } \\
\text { Name }\end{array}$ & Yes & No & Douglass, Neal & \\
\hline $\begin{array}{l}\text { Normalized } \\
\text { Name }\end{array}$ & Yes & No & douglass neal & $\begin{array}{l}\text { Machine generated by the } \\
\text { system, using NACO } \\
\text { transformation }\end{array}$ \\
\hline Name Type & Yes & No & Personal & $\begin{array}{l}\text { Options are Personal, } \\
\text { Organization, Event, Software, } \\
\text { Building }\end{array}$ \\
\hline $\begin{array}{l}\text { Biography/ } \\
\text { History }\end{array}$ & No & No & $\begin{array}{l}\text { May 15, 1988: Name changed } \\
\text { to University of North Texas }\end{array}$ & $\begin{array}{l}\text { Text is formatted in Markdown } \\
\text { with schema.org additions }\end{array}$ \\
\hline Begin Date & No & No & $1900-04-14$ & $\begin{array}{l}\text { Extended Date/Time Format } \\
\text { (EDTF) }\end{array}$ \\
\hline End Date & No & No & $1983-11-25$ & EDTF \\
\hline Disambiguation & No & No & Photographer & \\
\hline Record Status & Yes & No & Active & $\begin{array}{l}\text { Options are Active, Deleted, } \\
\text { Suppressed }\end{array}$ \\
\hline Merged With & No & No & & $\begin{array}{l}\text { Used to merge two records for } \\
\text { the same entity }\end{array}$ \\
\hline Variant Name & No & Yes & UNT & $\begin{array}{l}\text { Qualifier options are Acronym, } \\
\text { Abbreviation, Translation, } \\
\text { Expansion, Other }\end{array}$ \\
\hline Identifier & No & Yes & http://viaf.org/viaf/125477573 & $\begin{array}{l}\text { Qualifier options include } \\
\text { ORCID, VIAV, LCNAF, Wikipedia } \\
\text { Link, Google Scholar, Citations } \\
\text { Link, Homepage }\end{array}$ \\
\hline Note & No & Yes & & $\begin{array}{l}\text { Qualifier options are } \\
\text { Biographical/Historical, } \\
\text { Deletion Information, Non- } \\
\text { Public, Source, Other }\end{array}$ \\
\hline Location & No & Yes & $\begin{array}{l}33.206537000 \\
-97.1727100000\end{array}$ & $\begin{array}{l}\text { Qualifier options are Current } \\
\text { Location, Former Location }\end{array}$ \\
\hline
\end{tabular}

Table 2. Fields used in UNT Name App authority records. 
In addition to the support for a subset of MADS, we were interested in supporting the Schema.org vocabularies. These vocabularies have become one of the primary ways institutions are sharing linked data, by marking up existing human-readable HTML-based web pages with machine-readable tags; this allows the same content to be a part of both the LOD community and the greater Semantic Web. Finally, we wanted a system that we could use for a variety of name types including people, organizations, pieces of software, events and finally buildings. This flexibility would allow us to use the same system and framework for a wide variety of names that needed to be locally defined.

As we were developing this system we had in mind the five stars of open linked data that Tim BurnersLee enumerated in 2006 (see Figure 1).

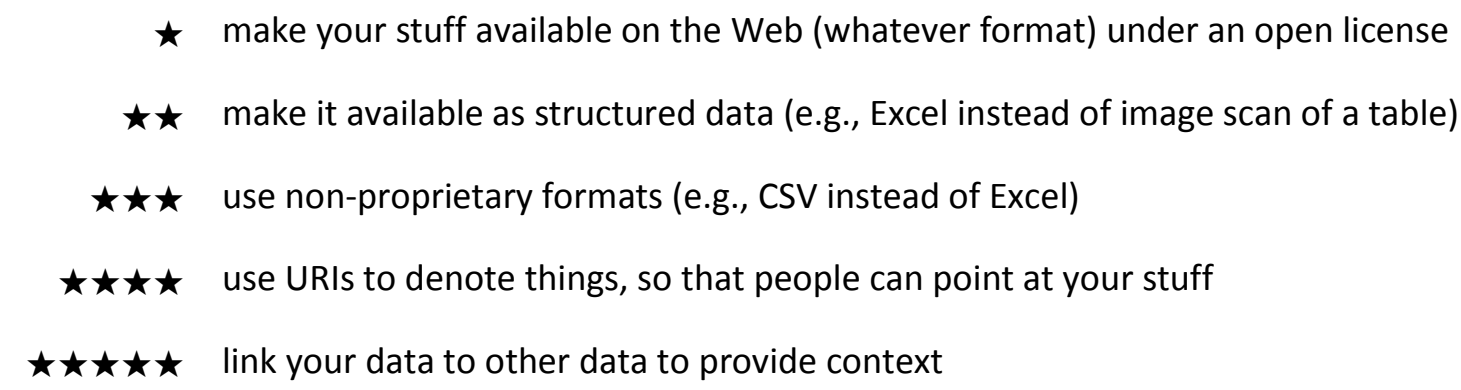

Figure 1. Summary of five stars of open linked data.

With an attempt to extend this five star approach, we decided that there were actually four steps that we would follow for establishing name authority in a staged approach:

1. Put vocabularies on the web as five-star open linked data.

2. Make vocabularies available to metadata creators who actively use them.

3. Store links, not strings.

4. Make data meaningful to users.

Stage one: building the app

In order to have a reasonable, long-term solution, the first step was to create a system for name authority that would meet all of the requirements of the five stars of open linked data. The system would need to accommodate names with descriptions as well as representations of relationships between an established name, our locally-created URI for the name and other web-based identifiers for the same entity. Additionally, we needed the ability to make this content available under a license that allowed for -- and encouraged -- reuse and incorporation into other systems; without any chance of linking back into our authorities, our system would most likely not succeed.

We decided to represent our local data model in a number of machine-readable formats including a native JavaScript Object Notation (JSON)-based representation, a record-based format using the MADS schema, and a version of the data using the Schema.org vocabulary and inline micro-data markup. Each name in the UNT Name App would have a unique identifier, used to globally identify a specific name from other names that might be similar. Finally, we made sure to incorporate mechanisms for the establishment of relationships between our locally-defined name authority record and other identifiers for the same name in other systems such as the Virtual International Authority File (VIAF), the Library of 
Congress Name Authority file (LCNAF) and web systems such as Wikipedia, Twitter and Google Scholar Citations, among others (see Figure 2). These components together meet the requirements of five-star data by putting information on the web in a structured format (stars 1-3), assigning URIs (star 4) and linking to other systems for context (star 5).

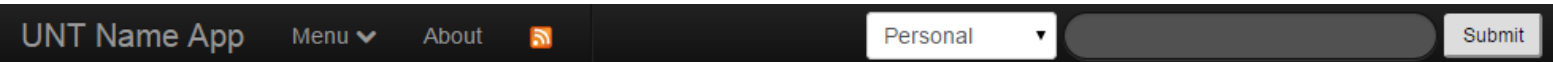

Abbas, June, 1964-

\begin{tabular}{|c|c|}
\hline Authorized: & Abbas, June, 1964- \\
\hline Name Type: & Personal \\
\hline URI: & http://digital2.library.unt.edu/name/nm0003231/ \\
\hline Date of Birth: & 1964 \\
\hline \multirow[t]{4}{*}{ Biographical Info: } & Affiliations \\
\hline & $\begin{array}{l}\text { - University of Oklahoma: Faculty } \\
\text { - State University of New York at Buffalo: Faculty } \\
\text { - University of North Texas: Alumna } \\
\text { - Emporia State University: Alumna }\end{array}$ \\
\hline & Subject Areas \\
\hline & $\begin{array}{l}\text { - User-centered digital libraries } \\
\text { - Institutional repositories } \\
\text { - Knowledge organization structures }\end{array}$ \\
\hline Links: & $\begin{array}{l}\text { - wr Internal: UNT Libraries Digital Collections } \\
\text { - } \text { I/ VIAF: http://viaf.org/viaf/122310895 } \\
\text { - } \approx \approx \text { LOC: http://id.loc.gov/authorities/names/n2010041548 } \\
\text { - } 8 \text { Google Scholar: http://scholar.google.com/citations?user=B-ozVCsAAAAJ\&hl=en } \\
\text { - in LinkedIn: http://www.linkedin.com/pub/june-abbas/14/288/b68 }\end{array}$ \\
\hline
\end{tabular}

\section{Alternate Formats}

\section{MADSIXML JSON}

Figure 2. Example record in the UNT Name App.

Stage two: making it available to metadata creators

Another important internal goal involved connecting the name authority files to the metadata creation process. Although the Name App was built in a way to make authority files and information externally visible and shareable, making tools available to metadata creators to incorporate name authority helps to ensure the use of established names. If metadata creators had to open a separate application to search for names, it would be harder to encourage continued authority control.

This stage was meant to provide a logical and straight-forward way of allowing our metadata creators to insert name authority into their daily workflow. We accomplished this by creating a JSON web service or application-programming interface (API) in the UNT Name App that would allow remote systems to easily query and use authorized values or even the links themselves. Then we updated our metadata system so that our metadata entry form would query the Name App any time an editor started to type 
within a designated name field (for creators, contributors, or publishers) and display possible matches (see Figure 3).

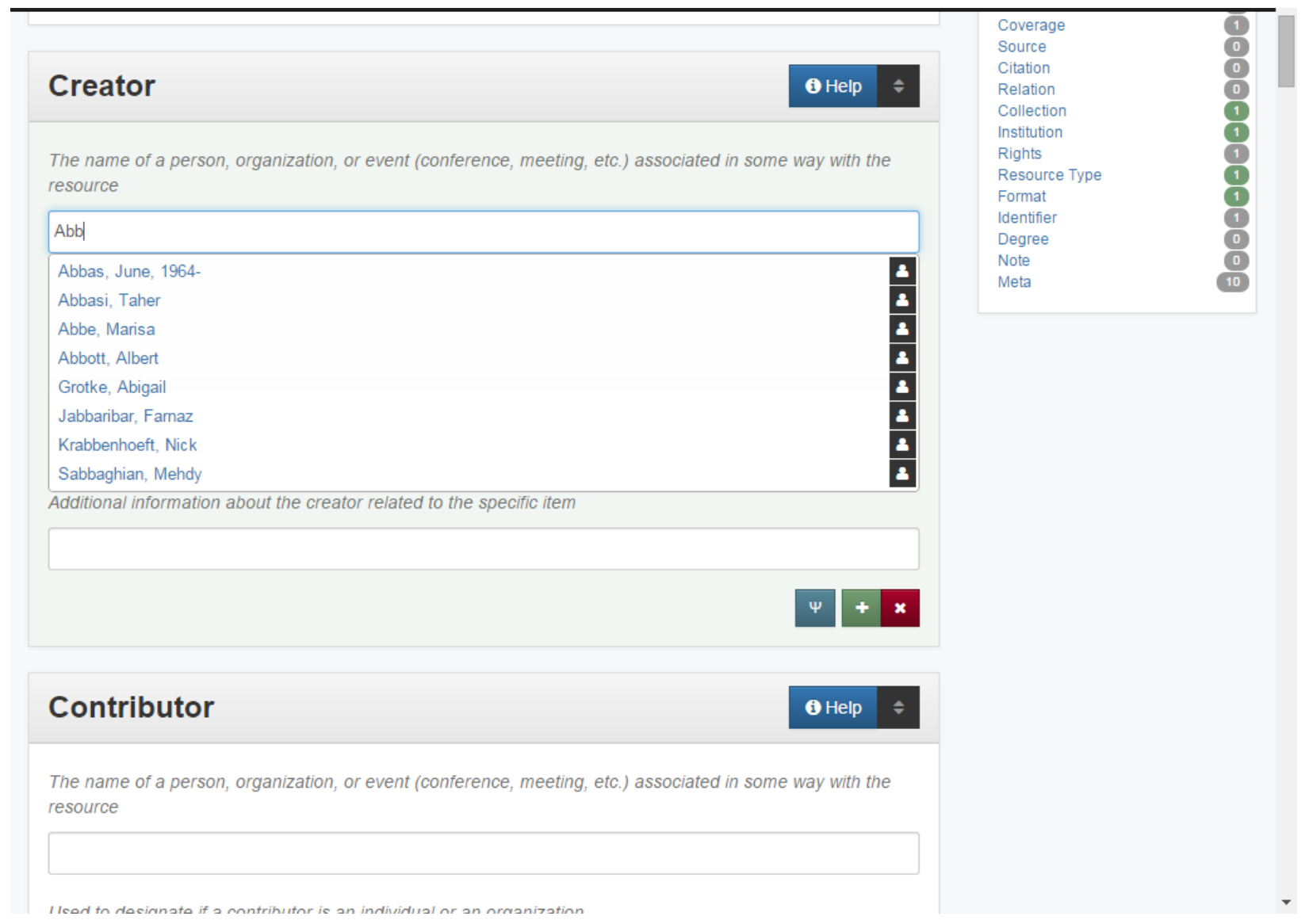

Figure 3. Example type-ahead options in the metadata editing system.

Stage three: storing links

The next stage, storing links, will require a significant change to the underlying infrastructure that we have not yet made. However, the goal is to embed machine-readable URIs in the metadata stored by the system while displaying human-readable names for users. This change is necessary because computers can read and compare strings, but they cannot distinguish when variations are all forms of the same name or different persons with similar names. As collections grow, there is an even greater chance that common or popular names will recur.

For example, we found a number of versions of the name Donald W. Smith (Don Smith, Donald Smith, Don W. Smith, etc.). A computer can easily compare these names and provide a probability that the names should all be the same, however it cannot verify that the names all belong to the same person, or necessarily choose the most appropriate "authorized" version -- which may even be different from the available combinations. In fact, the name entries are spread across two different collections, including reports published by the National Advisory Committee for Aeronautics (NACA) during the 1940s-1950s and UNT theses and dissertations from the 2000s. Since the UNT professor listed as a committee member on the ETDs was still completing his bachelor's degree around the time that the NACA reports 
were published, we can make an educated assumption that there are likely two Donald W. Smiths represented in our system: one who authored aeronautical engineering reports and a UNT biology professor. However, we reached this conclusion by considering multiple contextual factors that generally require human intervention.

At this point we have a secondary problem; we have two unique persons in our system, both represented by the name "Smith, Donald W." So long as we store strings, even if we have separate authority records for these two people, there is no way to denote in the record which Donald Smith we mean -- the text value is exactly the same. Similarly, there is no way for us to clearly express the fact that a name in a particular record has been verified and refers to "the first" Donald W. Smith, while the same name in another record might be the same person, but has not yet been verified. Theoretically, storing links when names have been verified would let us note which names have been actively controlled and eventually collocate materials by an individual without retrieving materials by another author with the same name.

Another potential benefit is the possibility of enabling functionality to connect all items by an author while displaying different forms of a name. For example, if we had a situation where we particularly wanted or needed to display the name as it appears on the item, we could store the link identifying the author to the system while displaying alternate text to users (see Figure 4).

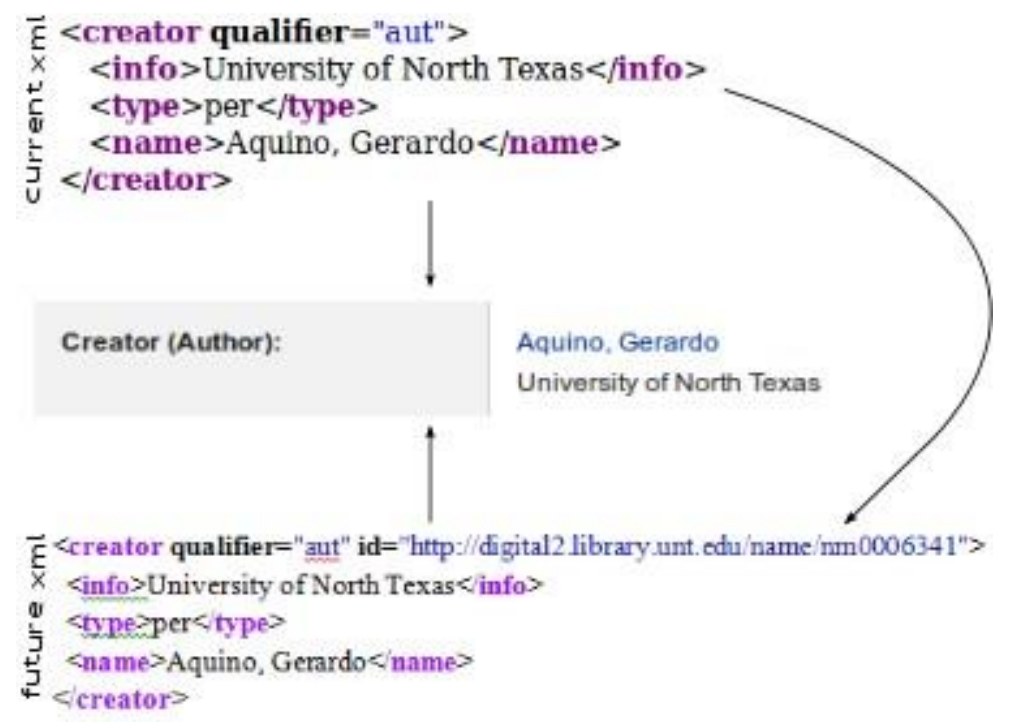

Figure 4. Example of metadata with name identifier links in the XML coding.

The figure above contains an example (at the top) of the current XML coding used in the UNT Digital Library for a creator in which the name and information/affiliation are stored as strings. Simple controlled vocabularies represent creator type (person) and role (author) and show up as codes in the XML. Beneath, a snippet of modified XML illustrates metadata with a unique URL identifier associated with that name stored in the coding. How the name displays to the user may not change -- shown in the center of the figure -- but the name could be displayed differently for different publications (e.g., as it appears on each item) while designating all records with the same URL name identifier as having the same creator or contributor. 


\section{Stage four: making names meaningful for users}

Ultimately, a primary goal of linked data and projects that make use of various kinds of metadata is to create a system or interface that is more meaningful for users. When information is linked, it provides options to explain context, improve search options, and create dynamic displays. In the commercial domain, companies have leveraged their resources to build specialized user interfaces around name authority, such as the Amazon author and artist pages, WorldCat Identities or Open Libraries author pages. These pages help users find information about particular resources, but also provide contextual information, which may include biographical information, publishing or recording history, or recommendations for finding similar items.

Unfortunately, local IRs do not generally have the resources or large data stores to create similar systems. However, the names controlled in academic IRs and similar institutions are often not managed or well represented outside of these organizations. This makes it even more important to expose name authority data and start building metadata that could be used to generate end-user functionality around academic names and names not represented in the commercial world.

\section{Implementation}

Once the Name App was built, we wanted to start compiling name authority records for entities in our system, even though the infrastructure and display pieces are ongoing. Until this point, names for scholarly materials had been entered in metadata records based on the version found on the item, since we did not have an efficient way of performing authority control. However, this led to retrieval issues that affected our campus and the wider research community using our items. Based on our primary goal of controlling institutionally-important names, we started with names in the UNT Scholarly Works collection and our collection of UNT Theses and Dissertations.

\section{Background on the collections}

UNT started requiring graduate students to submit digital copies of theses and dissertations in 1999, meaning that more than ten years of modern electronic theses and dissertations (ETDs) are available in the UNT Digital Library. For this collection, author names are unlikely to create discrepancies since most write only one thesis or dissertation, however, for each ETD we also document the student's committee members who signed off on the thesis or dissertation. Professors may serve on many different committees over time and their names are often represented in different ways, depending on how a student submits them. For example the professor Dr. William Moen may be referred to as Dr. Moen, Bill Moen, Dr. Bill Moen, Dr. William Moen, or in an extreme, Dr. Bill. Additionally, we have been actively working to scan and provide access to UNT theses and dissertations published in print, prior to 1999, creating an even larger pool of names and variations. In the case of current professors, there is also the likelihood that some of the names will have crossover between this collection and UNT Scholarly Works.

In 2010 the UNT Libraries decided to develop an institutional repository (IR) for the scholarly output of the faculty, staff and students at UNT. While institutional repositories were in no sense a novel idea, this represented the first time that the UNT Libraries would make an overt effort to solicit, collect and provide access to these materials. The IR would also assist in fulfilling the expectations of the UNT Open Access Policy passed by the UNT Faculty Senate and accepted by the UNT Regents as an official UNT 
Policy, encouraging UNT faculty and staff to deposit scholarly output. In practice, the UNT Scholarly Works IR is a collection within the greater UNT Digital Library.

Although staff members recognized the need for authority control within the IR, this was not a priority at the start of the collection, both because we did not have established mechanisms, and because there was a desire to show a quick amount of progress to demonstrate that the service was working as designed. This meant that authors' names might be entered under several variants if the versions of their names differed across publications, e.g., "Laura Waugh" and "L. Waugh." By having two values for one name, end-users (often the authors themselves) might not be able to retrieve all of the expected publications written by a particular person.

\section{Establishing name authority}

In 2013 the UNT Name App had been built and we had identified the pilot collections. As a test, we extracted all creator and contributor names from the UNT Scholarly Works and ETD collections. These names and their associated name types (either person or organization) were compiled into a list, sorted and simplified by removing duplicate values. After that we used the UNT Name App's ingest API to establish new authority records for each of the names on the list.

At this point, staff members spent time identifying and merging records that represented the same identity. As records in the UNT Name App were merged, the metadata values for associated resources in the UNT Digital Library were also corrected manually, to match the authorized form of the name. When we finished with this set of names (over 3,000 in total) we considered the data "cleaned" and we were then able to run reports. This also let us experiment with new ways of interacting with the data that previously unable to do because of the inconstancy of the data in the system.

Today when we receive new resources for either the UNT Scholarly Works repository or the UNT Theses and Dissertations collection we add the names to the UNT Name App to establish them in the system before we create the bibliographic metadata for the item. This way we are able to use the authorized version for each name via the metadata entry screen. While initially time-consuming, the effort of creating new name authority records after the original loading of names has been minimal, with the addition of only a few dozen new names each month.

\section{Discussion}

Overall, the implementation of name authority in our collections has been successful. Although we do not plan to attempt system-wide control of all names in our collections, we now have the ability to establish authority records that we deem important for any project. For example, the Texas Fashion Collection has a large number of names that are frequently entered in metadata. Using authority records for the names in this collection would make metadata entry more streamlined and allow for contextual linking since some of the designers may have established authorities in other systems. Additionally, we have started using the Name App to better define current and historic names for buildings on the UNT campus, which is useful for some of the local archival collections we are digitizing that document UNT's history. 
We have found that incorporating name authority control into existing digital library collections can be accomplished in a straightforward and sustainable way. The ability to integrate authorized names during the metadata entry process has helped to streamline this process and is directly related to basing our authority work on a framework that supports a connection between authorities and metadata records. One outcome of this work that we did not expect was the interest from many other institutions around the country in doing this same sort of work with their local names; once they heard about our UNT Name App, several of them wanted to install the tool for their local institutions. Initially, we were not ready to make the code publicly available, but in early 2015 we released the source code under an Open Source License on the UNT Libraries' Github site under the project name "django-name." Hopefully by making this code available, others will be able to repeat, modify and improve upon the process that we used.

\section{Future Steps}

There is a continuing need in libraries and other cultural heritage organizations -- such as archives and museums -- to engage in name authority activities. Although the wide range of existing name authority projects demonstrates recognition of this need and the connection to functionality for end users, the diversity in this area also shows the need for concerted efforts on multiple fronts to make headway on such a huge task. Even with various national and international efforts, there is still a need for local institutions to manage and curate names that are important to the life cycle of information resources in our academic institutions. However, to ensure that these efforts will have the largest impact and be compatible with other projects, we should do our best to make sure that contributions to name authority work meet standards and expectations within the wider community, so that users at other institutions or outside the library sphere can take advantage of the work. By starting with the five stars of open linked data and then using an iterative approach, such as the one described in this chapter, we can capitalize on local efforts to create a cooperative compilation that can be shared openly and continue to grow well into the future.

\section{References}

Berners-Lee, Tim (2006, July 7) Linked Data, www.w3.org/Designlssues/LinkedData.html

Ilik, V. (2014) Cataloger Makeover: Creating Non-MARC Name Authorities, Cataloging \& Classification Quarterly, 53(3-4), 382-398

OCLC (2012, June 20) OCLC adds linked data to WorldCat.org, www.oclc.org/news/releases/2012/201238.en.html

OCLC (2012, December 7) VIAFbot edits 250,000 Wikipedia articles to reciprocate all links from VIAF into Wikipedia, www.oclc.org/research/news/2012/12-07a.html

OCLC (2015) VIAF: Virtual International Authority File, www.oclc.org/viaf.en.html

Salo, D. (2009) Name Authority Control in Institutional Repositories, Cataloging \& Classification Quarterly, 47(3-4), 249-261

Tarver, H., Waugh, L., Phillips, M., \& Hicks, W. (2013) Implementing Name Authority Control into Institutional Repositories: A Staged Approach, http://digital.library.unt.edu/ark:/67531/metadc172365/ 
Texas A\&M University Libraries (2015, April 14) ORCID and Other Researcher Identifiers, http://guides.library.tamu.edu/content. php?pid=553864\&sid=4564757

Tillett, B. B. (1989) Considerations for Authority Control in the Online Environment, Cataloging \& Classification Quarterly, 9(3), 1-11

University of North Texas Libraries (2015) Input Guidelines for Descriptive Metadata, www.library.unt.edu/digital-projects-unit/input-guidelines-descriptive-metadata

\section{Websites}

1. Library of Congress Name Authority File, http://id.loc.gov/authorities/names.html

2. Virtual International Authority File (VIAF), https://viaf.org

3. Open Researcher and Contributor ID (ORCID), http://orcid.org

4. International Standard Name Identifier (ISNI), www.isni.org

5. The Portal to Texas History, http://texashistory.unt.edu

6. UNT Digital Library, http://digital.library.unt.edu

7. The Gateway to Oklahoma History, http://gateway.okhistory.org

8. UNT Vocabularies, http://digital2.library.unt.edu/vocabularies

9. UNT Name App, http://digital2.library.unt.edu/name

10. Metadata Authority Description Schema (MADS), www.loc.gov/standards/mads

11. Schema.org, http://schema.org

12. Google Scholar Citations, https://scholar.google.com/intl/en-US/scholar/citations.html

13. Amazon, www.amazon.com

14. WorldCat Identities, www.worldcat.org/identities

15. Open Library: Authors, https://openlibrary.org/authors

16. UNT Open Access Policy, http://openaccess.unt.edu/unt-open-access-policy

17. Texas Fashion Collection, http://digital.library.unt.edu/explore/collections/TXFC

18. UNT Name App source code, https://github.com/unt-libraries/django-name 